\title{
Fully Automated Machine for Scanning SiPM detectors
}

\author{
A. Rychter*, J. Marzec, G. Domanski, M. Dziewiecki, B. Konarzewski, R. Kurjata, K. \\ Zaremba, M. Ziembicki \\ Warsaw University of Technology, Institute of Radioelectronics and Multimedia Technology, \\ Warsaw 00-665, Poland \\ E-mail: arychter@ire.pw.edu.pl
}

\begin{abstract}
A fully automated measurement system has been built to measure uniformmity of the parameters of SiPM detectors with very high pixel densities. It has a light source with a microfocus spot (FWHM $=3 \mu \mathrm{m}$ at the detector surface) and a precise positioning system (1 $\mu \mathrm{m}$ accuracy). A temperature control system with water cooling has been used to set the temperature of the measured detector. Finally, a low noise, custom made front-end amplifier has been designed to receive SiPM signals. This paper presents the MAPD-3N detector measurement results - uniformity of gain and relative photon detection efficiency (PDE).
\end{abstract}

International Conference on New Photo-detectors, PhotoDet2015

6-9 July 2015

Moscow, Troitsk, Russia

${ }^{*}$ Speaker. 


\section{Introduction}

This paper presents a dedicated measurement system designed for measuring parameter uniformity of Silicon Photomultipliers (SiPM). SiPMs are rapidly developing photosensitive devices which are already used as an alternative to photomultipliers. An innovative SiPM detector, Micropixel Avalanche Photodiode (MAPD) from Zecotek has been measured. The MAPD detector utilizes a technology of deeply buried microwells [1] [2], which offers improved photon detection efficiency, high response linearity and high pixel density [3]. The MAPD detector does not have quenching resistors nor metallization grid on the sensor's surface, thus the fill factor should be nearly $100 \%$ regardless of pixel density[2]. This paper presents results of gain and PDE uniformity measurements for MAPD-3N detector. MAPD-3N detector is $3 \times 3 \mathrm{~mm}^{2}$ device with 135000 pixels ( $8 \mu \mathrm{m}$, pitch), the PDE is $30 \%$ and the electron gain of the detector is in range of $10^{4}$ to $10^{5}$ [2][4].

\section{Measurement System}

Using the designed system, it is possible to measure parameters such as gain and relative PDE as functions of the position on the detector surface and thus it allows to estimate uniformity of the parameters for the detector. For every position of a pulsed light source a histogram of signal amplitudes is built, from which various parameters are calculated. A single scan of the detector surface consists of the following stages:

1. Set working parameters: operating voltage, temperature, light level and pulse frequency.

2. Perform fast a scan of signal amplitude with a rough step and calculate boundary coordinates of the detector's surface.

3. Choose a region of interest and perform light beam focusing in the middle of this region.

4. Scan the desired region: collect histograms of amplitudes for every point of the scan.

5. Calculate and plot parameters. Analyse their uniformity.

\subsection{Front-end Electronics and Data Acquisition}

For the amplitude-histogram-based measurements the signal to noise ratio (SNR) has to be high enough to distinguish the positions and heights of the histogram peaks. The equivalent noise charge (ENC in electrons) has to be much lower than the detector's electron gain for desired working conditions (temperature and bias voltage). SiPM detectors with very high pixel densities have relatively low electron gain due to small pixel sizes, thus a custom made charge sensitive amplifier (CSA) has been designed. The simplified schematic of the front-end electronics and data acquisition system is shown in figure 1.

The front-end electronics consists of a low noise CSA and an active RC shaping circuit to fit $200 \mathrm{~ns}$ integration window of the charge to digital converter - QDC V965. The charge to digital converter is triggered with a pulse generator and sends its data via VME and further USB to a computer. The computer software gathers data and builds histograms. For every histogram a set of parameters can be calculated. 


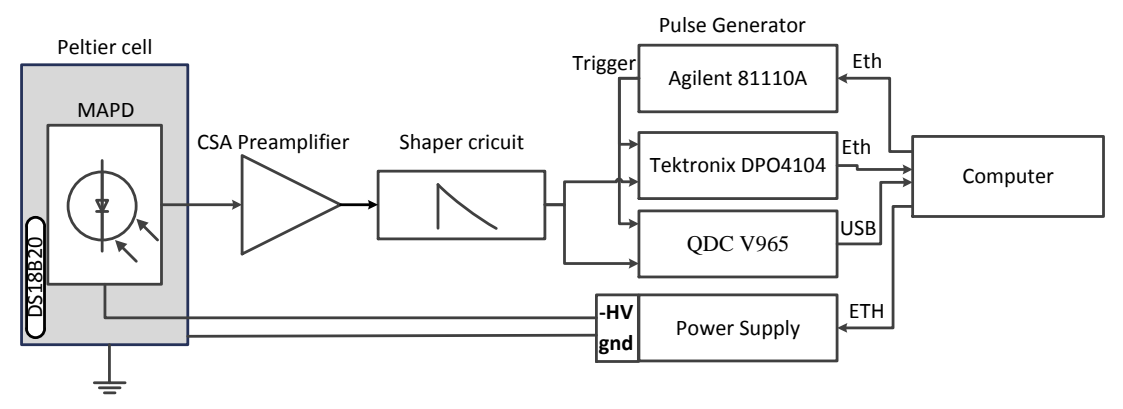

Figure 1: Schematic of the front-end and data acquisition system.

\subsection{Light Source and Positioning System}

The measurement of uniformity of parameters requires a special device for positioning and focusing the light spot on the surface of the detector. A system for light positioning was developed, a schematic is presented in Figure 2. The device consists of a custom made PCB for controlling three stepper motors (Thorlabs ZST213b) driving a micrometer block (Thorlabs RB13M), which enables positioning of a collimator and a microfocus lens in three dimensions with precision of $1 \mu \mathrm{m}$ over $13 \mathrm{~mm}$ span. The alignment of the detector relative to the scanning axis is accomplished manually and can be corrected after the first scan. A picosecond laser controller PiLas EIG2000DX with two laser diode modules of wavelengths of $370 \mathrm{~nm}$ and $440 \mathrm{~nm}$ is used as a light source. There is no possibility to monitor the light intensity during the scan, although tests showed very good stability of the PiLas system. The FWHM of the light spot is dependent on the distance between the microfocus lens and the surface of the detector. The measurement of the FWHM of the light spot was performed with a CCD matrix (pixel size $1.4 \mu \mathrm{m}$ ) inserted in the place of the detector. The measured lowest FWHM is around $3 \mu \mathrm{m}$. The FWHM of $100 \mu \mathrm{m}$ can be achieved if the microfocus lens is moved $400 \mu \mathrm{m}$ away from its focal position.

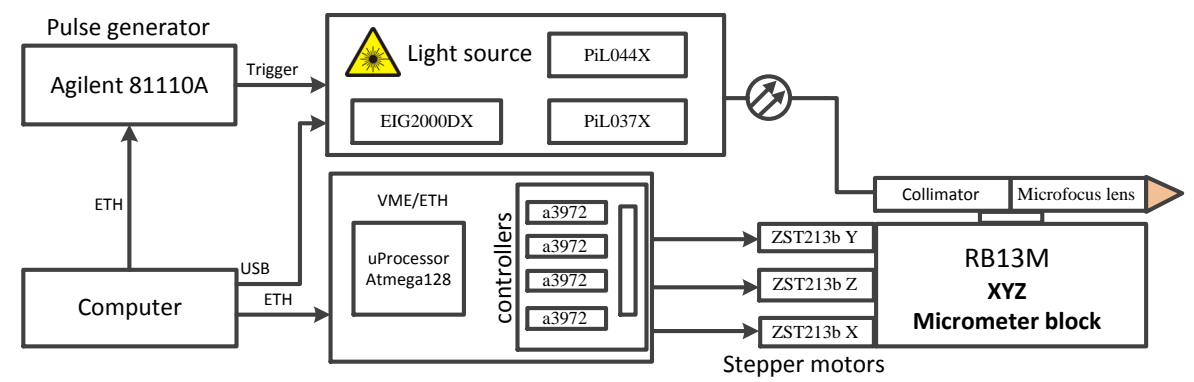

Figure 2: Schematic of the light source and the light spot positioning system.

A program developed in Matlab environment communicates with both the light source and the positioning system. It is possible to set the light level and pulse frequency. The laser controller is 
triggered with a pulse generator - Agilent $81110 \mathrm{~A}$. The same signal is used as a start condition in DAQ system.

\subsection{Temperature Control System}

The temperature control system has been realized with a proportional-integral (PI) controller running as a Matlab script. A schematic of the temperature control system is presented in Figure 3. The SiPM detector is placed on a metallic plate which is cooled with a Peltier cell. The tem-

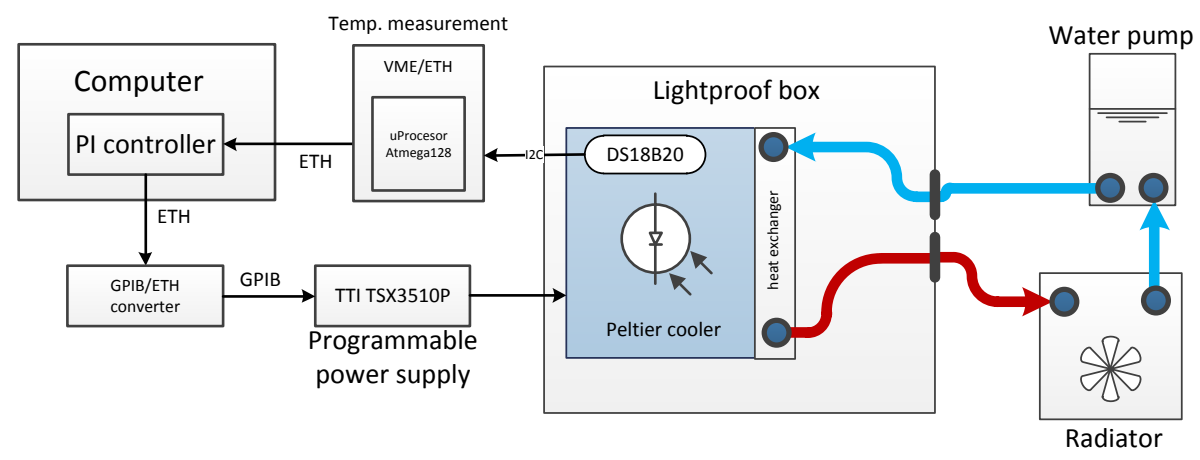

Figure 3: Schematic of a temperature control system.

perature of the detector is measured with a DS18B20 sensor. Power supply of the Peltier cell (TTI TSX3510P) is remotely controlled via Ethernet network connection. The heat produced by the Peltier cell is transferred outside the lightproof box with a special water cooling system consisting of a heat exchanger, a water pump and a radiator with a fan. The system allows controlling the temperature of the detector in a range from $0{ }^{\circ} \mathrm{C}$ to $25^{\circ} \mathrm{C}$ with an accuracy of $0.5^{\circ} \mathrm{C}$.

\section{Measurement Results}

In this section results of measurements of one sample of the MAPD-3N detector will be shown. All scans have been performed with a constant temperature, bias voltage and laser settings:

- temperature: $16^{\circ} \mathrm{C}$

- operating voltage: $89 \mathrm{~V}$

- electron gain $\approx 6.2 \times 10^{4}$

- laser wavelength: $440 \mathrm{~nm}$

- pulse frequency: $100 \mathrm{kHz}$

\subsection{Initial Fast Scan of Signal Amplitude}

The first scan of the MAPD-3N has been performed while measuring only the signal amplitude. The scan step has been set to $100 \mu \mathrm{m}$. For every position of the laser spot, signal has been measured with an oscilloscope and sent to the computer. The average number of fired pixels was 
50. The FWHM of laser spot was $100 \mu \mathrm{m}$. The result of the signal amplitude scan is presented in Figure 4. The scan has been performed in full range of $13 \mathrm{~mm}$ in $\mathrm{X}$ and $\mathrm{Y}$ planes. The result gives information about position of the detector's active area. The centre position of the detector is $x \approx 3 \mathrm{~mm}, y \approx 7 \mathrm{~mm}$. An anode connection pad is visible in the left upper corner of the detector.
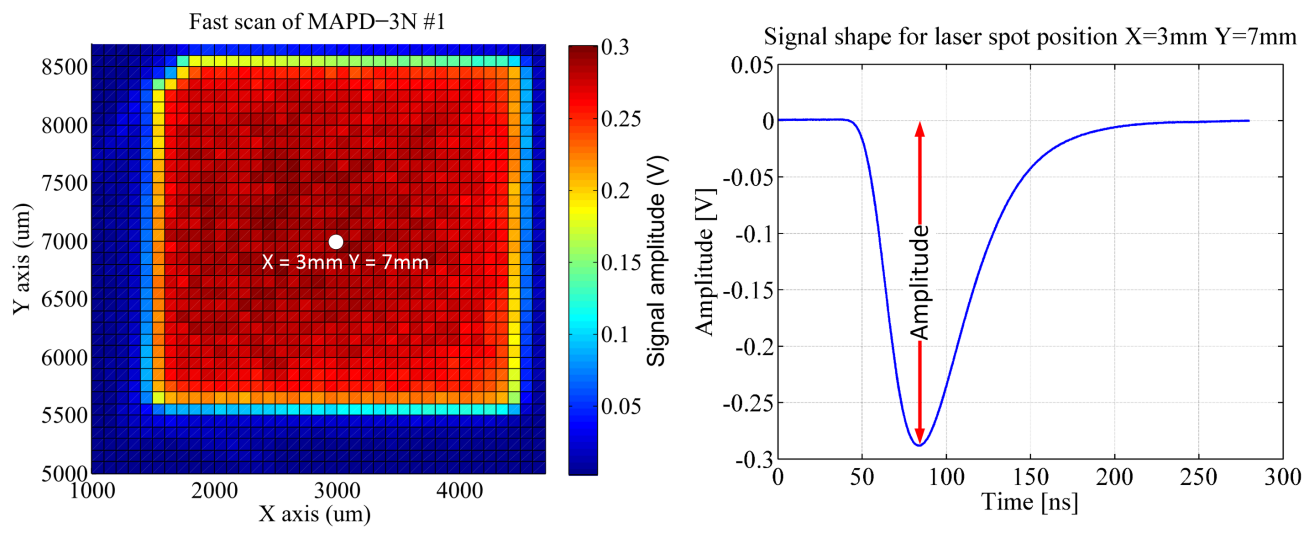

Figure 4: Fast scan of signal amplitude of MAPD-3N detector to the left. Typical waveform signal to the right.

\subsection{Rough Scan with Histogram Measurements}

After the fast scan of a signal amplitude, a scan with histogram collection within limited boundaries and the same step of $100 \mu \mathrm{m}$ has been performed. For every position of the laser beam a histogram of signal charge with 100k samples has been recorded. A pulse frequency has been set to $100 \mathrm{kHz}$, so a collection of a single histogram took around $1 \mathrm{~s}$. The average number of fired pixels was 2 .

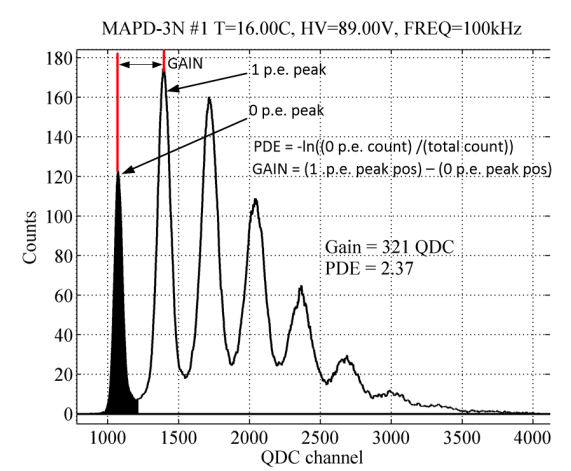

Figure 5: An example of a collected histogram. For the gain measurement the distance between 0 and 1 photoelectron peak positions is used. The relative PDE measurement is based on the number of collected 0 p.e. and the total counts of the histogram.

Results of relative PDE and a gain calculation are presented in Figure 6. On the left plot the relative PDE is presented as a mean number of fired pixels. On the right plot a gain measurements are shown. The alignment of the chip looks a little bit different from Figure 4; the reason for that is a movement of the chip between the measurements. The gain value outside the chip (calculated 
from signal from uniformly distributed dark pulses) is higher because it is not affected by long recovery time of the detector. Comparing two of these plots, it can be seen that uniformity of both parameters looks similar. The gain values drop significantly on left edge of the detector surface. For the corresponding area the mean number of fired pixels is also lower. Lower values of the gain and relative PDE (within the chip area) is implied by the fact that a breakdown voltage is higher for these areas while the operating voltage $(89 \mathrm{~V})$ stays the same. It can bee seen that there are several outlying points, the reason for that may be local scratches or dust particles on the top of the detector window which result in lower signal value. These points are not visible in Figure 4 due to a different measurement technique and a defined colormap range of the plot.
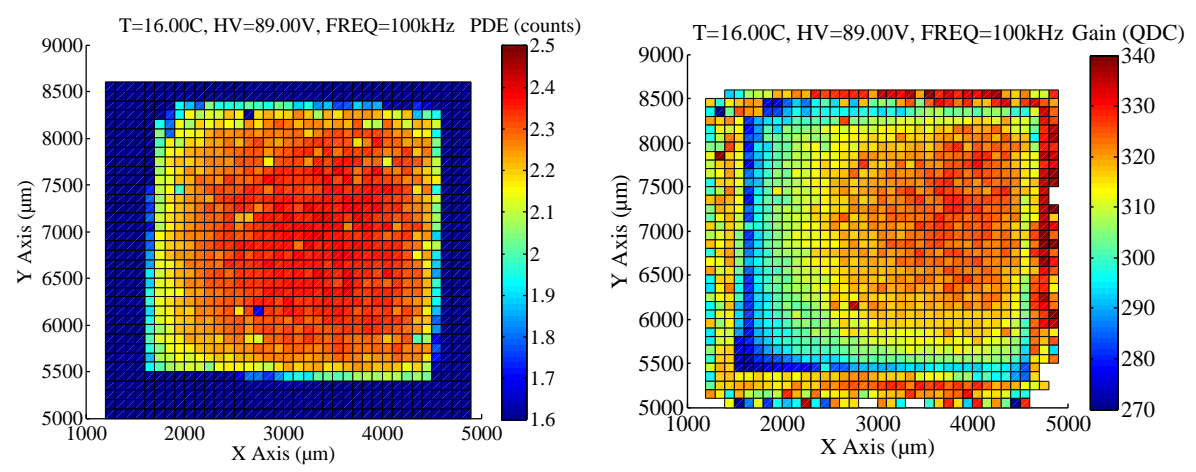

Figure 6: The result of MAPD-3N detector scan with a histogram collection. Left plot: distribution of relative PDE. Right plot: gain measurement.

\subsection{Fine Scan with Histogram Measurements}

The second scan of MAPD-3N detector has been performed with a fine step of $1 \mu \mathrm{m}$. The field of scan was $70 \times 70 \mu \mathrm{m}$. For this scan a light beam has been focused on a detector surface to a size of $3 \mu \mathrm{m}$. The average number of fired pixels was 1.5. It can be seen in Figure 7 that the 0 p.e. peak has lower amplitude when the light spot is positioned in the centre of the micro cell, which means that the relative PDE is a little bit higher in this point.

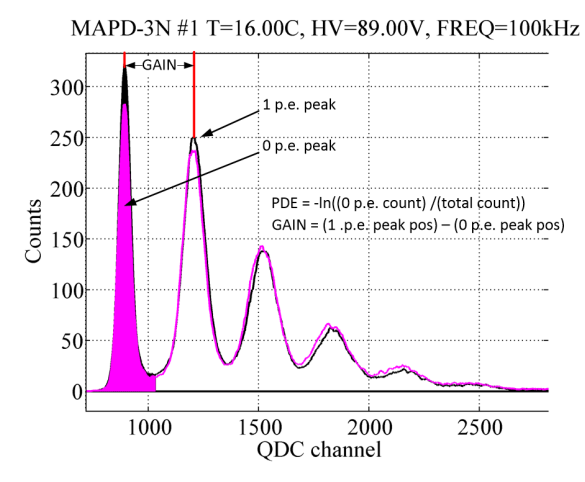

Figure 7: Two examples of collected histograms. The pink one is taken for light spot positioned in the centre of a micro cell, the black one at the boundary.

The results of the fine scan are shown in Figure 8. On the left plot relative PDE is presented, positions of buried microwells are visible. The value of the relative PDE for positions above the 
microwells is around $15 \%$ higher when comparing to the position between the microwells. On the right plot the gain distribution is presented. The gain variation are caused by the effects of the recovery time. When light illuminates the centre of the pixel, the gain value is a little bit lower. When neighbouring pixels "share" the light beam, the recovery time influence on gain is weaker.
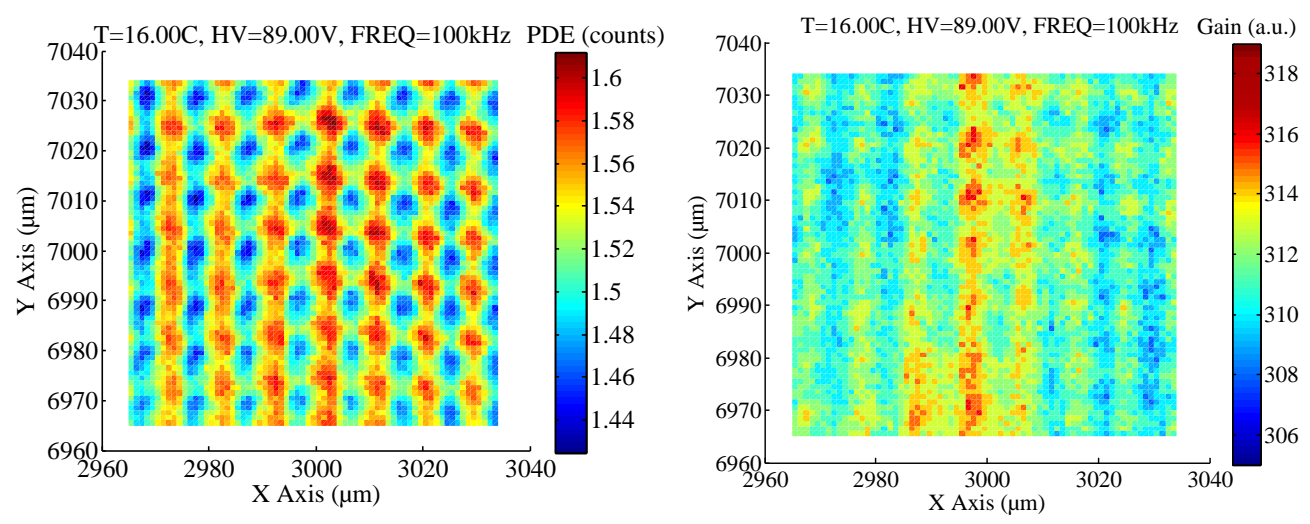

Figure 8: Results of MAPD-3N detector scan with a histogram collection. On the left plot - distribution of the relative PDE. On the right plot gain measurement.

\section{Conclusions}

A dedicated measurement system for measuring the uniformity of parameters of SiPM detectors has been build. A sample of MAPD-3N detector has been measured. Results of measurements showed non-uniformity of gain and relative PDE especially in areas close to edges of the detector.

\section{Acknowledgement}

This work is supported by the Polish National Science Centre grant DEC-2011/01/M/ST2/02350.

\section{References}

[1] Z.Ya. Sadygov, Russian Patent 2316848 of 01.06 .2006

[2] Zecotek MAPD White Paper, Zecotek, 2011

[3] A. Rychter, Simulation and Measurement of Super-High Density Micropixel Avalanche Photodiodes, Proc. of SPIE Vol. 8454, 2012 SPIE.

[4] N. Anfimov, I. Chirikov-Zorin, A. Dovlatov, O. Gavrishchuk, A. Guskov, N. Khovanskiy, Z. Krumshtein, R. Leitner, G. Meshcheryakov, A. Nagaytsev, A. Olchevski, T. Rezinko, A. Sadovskiy, Z. Sadygov, I. Savin, V. Tchalyshev, I. Tyapkin, G. Yarygin, and F. Zerrouk. Novel micropixel avalanche photodiodes (MAPD) with super high pixel density. Nuclear Instruments and Methods in Physics Research A, 628:369 371, February 2011.

[5] D. Renker. Geiger-mode avalanche photodiodes, history, properties and problems. Nuclear Instruments and Methods in Physics Research Section A: Accelerators, Spectrometers, Detectors and Associated Equipment, 567(1):48-56, 2006. 